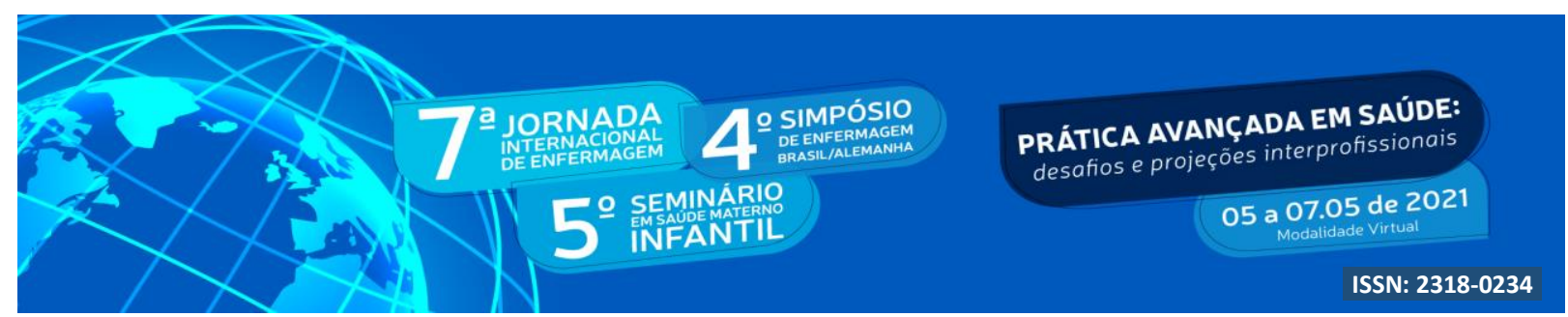

DOI: http://doi.org/10.48195/jie2021-006

\title{
PREENCHIMENTO E INTERPRETAÇÃO DO BALANÇO HÍDRICO EM UNIDADE DE TERAPIA INTENSIVA ${ }^{1}$
}

\author{
Ana Elza Oliveira de Mendonça²; Isabel Pires Barra ${ }^{3}$; Júlia Fonseca dos Anjos ${ }^{4}$; \\ Micheline Veras de Moura5; Dândara Nayara Azevêdo Dantas'
}

\begin{abstract}
RESUMO
Objetivou-se destacar aspectos relevantes para o preenchimento e interpretação dos resultados do balanço hídrico em pacientes críticos. Trata-se de um artigo de atualização, realizado por meio da análise de publicações e de reflexões oriundas da prática clínica assistencial de pacientes adultos em cuidados críticos na unidade de terapia intensiva. O preenchimento do balanço hídrico é uma atribuição da equipe de Enfermagem, realizado a cada duas horas, com cálculo das parciais a cada plantão e fechamento a cada 24 horas. Destacam-se como principais vias de entrada de líquidos no organismo a parenteral e oral, e de saída a diurese, perdas gastrintestinais e as drenagens. O resultado persistentemente positivo potencializa complicações cardiovasculares e parece estar relacionado com aumento da morbimortalidade. O balanço hídrico deve ser equilibrado e o resultado analisado concomitante com dados clínicos e exames laboratoriais e de imagem, para nortear a implementação de cuidados visando a segurança do paciente.
\end{abstract}

Palavras-chave: Balanço Hídrico; Unidade de Terapia Intensiva; Cuidados de Enfermagem.

\begin{abstract}
The objective was to highlight relevant aspects for completing and interpreting the water balance results in critically ill patients. This is an update article, carried out through the analysis of publications and reflections from the clinical care practice of adult patients in critical care in the intensive care unit. The filling of the water balance is an attribution of the Nursing team, carried out every two hours, with calculation of the partials at each shift and closing every 24 hours. Parenteral and oral fluids are the main pathways for fluids to enter the body, and diuresis, gastrointestinal losses and drainage are the main ways out. The persistently positive result offers the potential for cardiovascular complications and seems to be related to increased morbidity and mortality. The water balance must be balanced and the result analyzed concomitantly with clinical data and laboratory and imaging exams, to guide the implementation of care aimed at patient safety.
\end{abstract}

Key Words: Water Balance; Intensive Care Units; Nursing care.

\footnotetext{
${ }^{1}$ Artigo de atualização.

${ }^{2}$ Professora do Departamento de Enfermagem. Universidade Federal do Rio Grande do Norte. E-mail: anaelzaufrn@gmail.com

${ }^{3}$ Estudante do Curso de Graduação em Enfermagem. Universidade Federal do Rio Grande do Norte. E-mail: barraisa20@gmail.com

${ }^{4}$ Estudante do Curso de Graduação em Enfermagem. Universidade Federal do Rio Grande do Norte. E-mail: julia.sanjos18@gmail.com

${ }^{5}$ Doutoranda em Enfermagem. Universidade Coimbra. E-mail: michelineverasenf@yahoo.com

${ }^{6}$ Professora do Departamento de Enfermagem. Universidade Federal do Rio Grande do Norte. E-mail: dandara_dantas@hotmail.com 


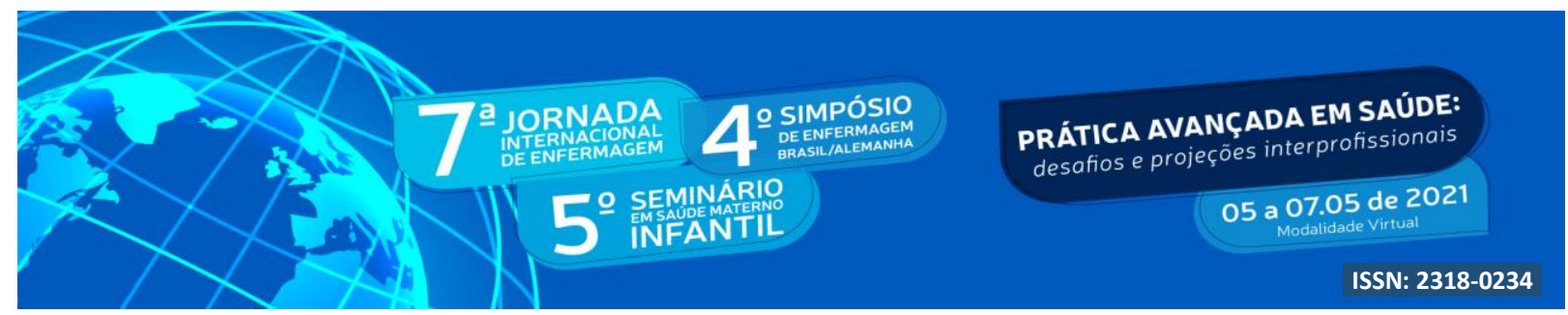

\section{INTRODUÇÃO}

A sobrecarga de fluidos é frequente em pessoas internadas em Unidades de Terapia Intensiva (UTI). A administração constante de fluidos, presença de lesão renal aguda e extravasamento vascular, devido a resposta inflamatória na sepse, contribuem para o seu desenvolvimento (ESPINOSA-ALMANZA ET AL., 2020).

Estudos mostram que o excesso de fluidos está associado ao aumento das taxas de mortalidade (SANTOS ET AL., 2020; OGBU; MURPHY; MARTIN, 2015). Desse modo, os fluidos intravenosos precisam ser dosados de forma adequada para reduzir o risco de danos à pessoa que já está gravemente enferma. Para isso, a realização do Balanço Hídrico $(\mathrm{BH})$ é fundamental para detecção precoce de sinais de retenção de líquidos e sobrecarga circulatória, especialmente em pacientes com comprometimento cardiovascular (LINHATES ET AL., 2016).

$\mathrm{O}$ BH integra a rotina de cuidados de Enfermagem em UTI, e destina-se ao monitoramento dos fluidos administrados e eliminados pelos pacientes críticos. A palavra "equilíbrio" é derivada do Latim equilibrium, na qual L.aequus significa igual + libra, balança. O equilíbrio hídrico é obtido quando há simultaneamente, o suprimento de líquidos de acordo com as necessidades metabólicas do organismo, aliado ao funcionamento adequado dos sistemas cardiovascular, pulmonar, digestório e renal. Nesse sentido, o BH desejável resulta do equilíbrio entre líquidos infundidos e eliminados (SERKOVÁ; MARECKOVÁ, 2019; LUCENA ET AL., 2017).

Estudos apontam uma fragilidade no registro do BH nas unidades de cuidados críticos, como o registro incompleto dos líquidos infundidos e/ou eliminados, assim como erros no cálculo do balanço, o que pode interferir na avaliação dos pacientes e, consequentemente, nas condutas terapêuticas (GUMS; SOUZA; SOUZA, 2021; GOMES ET AL, 2018).

Diante disso questiona-se: Quais aspectos são relevantes para o preenchimento e interpretação dos resultados do $\mathrm{BH}$ em pacientes críticos? Justifica-se a realização do presente estudo frente a relevância da temática para os profissionais que atuam em terapia intensiva 


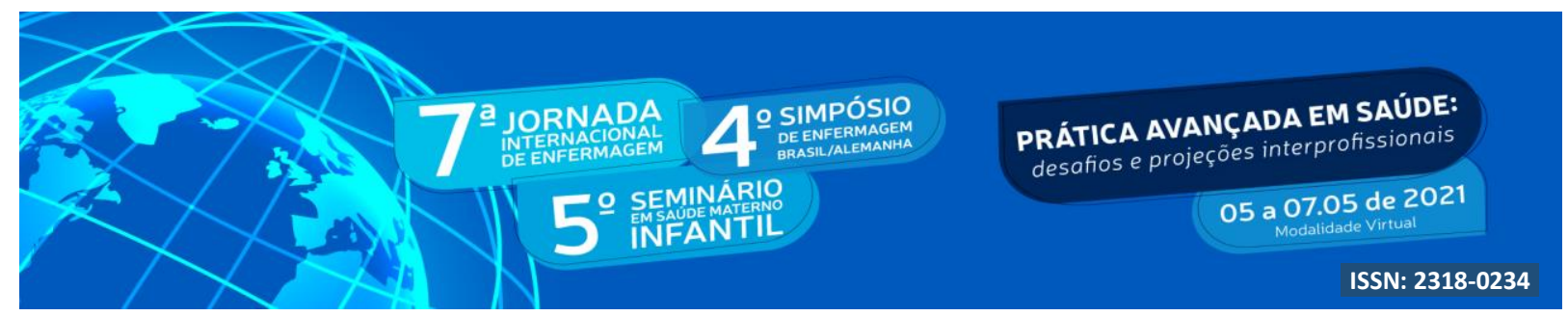

e a escassez de pesquisas que abordem a aplicabilidade do BH na prática clínica.

\section{OBJETIVO}

Destacar aspectos relevantes para o preenchimento e interpretação dos resultados do balanço hídrico em pacientes críticos.

\section{METODOLOGIA}

Trata-se de um artigo de atualização, que foi desenvolvido com base na definição do tema e objetivo, levantamento da literatura disponível em bases de dados científicas, leitura minuciosa dos títulos e resumos, seleção dos materiais pertinentes, definição da estrutura e seções a serem apresentadas nos resultados e elaboração da conclusão (POLIT; BECK, 2011).

As buscas foram realizadas em dezembro de 2020 nas bases de dados indexadas na Biblioteca Virtual em Saúde, utilizando-se os seguintes Descritores em Ciências da Saúde: "Balanço Hídrico" AND "Enfermagem”. Foram incluídos artigos completos disponíveis em inglês, português e espanhol. Inicialmente não foi realizado recorte temporal devido ao número reduzido de estudos sobre o tema nas fontes consultadas. Contudo, por tratar-se de um artigo de atualização optou-se por não incluir artigos com 10 anos ou mais de publicação.

A análise foi do tipo descritiva, realizada após leitura minuciosa dos artigos na íntegra, o que possibilitou a organização dos resultados em duas categorias, que esclarecem aspectos relevantes da prática assistencial de Enfermagem para o preenchimento e interpretação do resultado do BH. É importante destacar que, foram seguidos os preceitos éticos da pesquisa e todos os autores utilizados no desenvolvimento do artigo, foram devidamente citados no corpo do texto e nas referências em observância aos direitos autoriais dos pesquisadores.

\section{RESULTADOS E DISCUSSÃO}

Para facilitar a apresentação dos aspectos relevantes para realização do BH em pacientes críticos, foram criados os seguintes tópicos: Distribuição dos fluidos no organismo, 


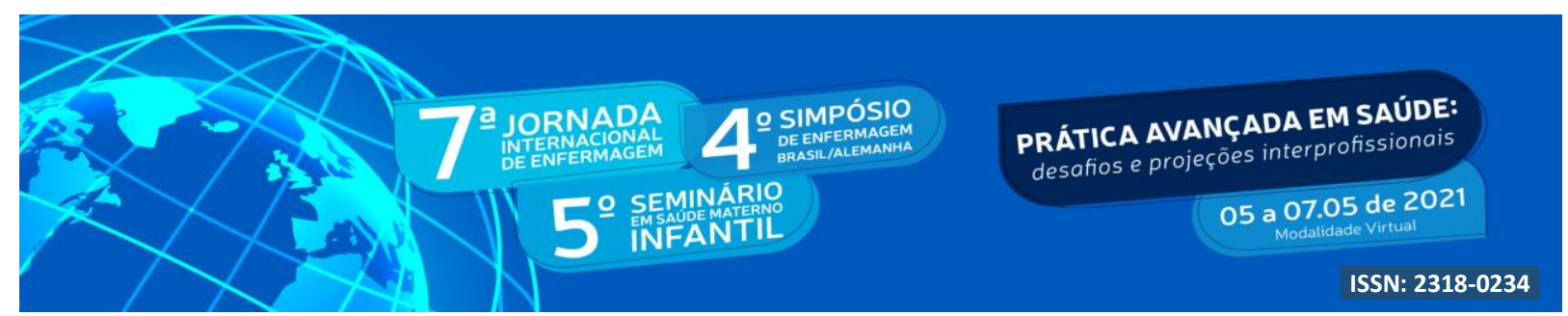

preenchimento do BH e aplicação prática do BH na UTI.

\section{Distribuição dos fluidos no organismo}

Os líquidos estão presentes em todas as células do corpo humano, com variações resultantes das constituições iônicas e do movimento de solutos, eletrólitos e diferentes gradientes de pressão dos compartimentos do organismo. A movimentação dos líquidos ou fluidos dentro do corpo acontece por meio da difusão, osmose e ou pressão hidrostática (MALNIC, 2009).

A primeira grande divisão dos líquidos no organismo consiste em dois grandes compartimentos: intra e extracelulares. Sabe-se que o líquido intracelular corresponde a aproximadamente $50 \%$ do peso corporal, com variações de acordo com o sexo, faixa etária e nível de hidratação. O líquido extracelular inclui o plasma, fluidos intersticiais (líquido que circunda as células em diferentes tecidos) e fluidos transcelulares (líquidos contidos no sistema digestório, cavidades pleural e peritoneal) (MALNIC, 2009).

A necessidade de água no organismo de um adulto pode ser calculada com base no peso corporal, aplicando a seguinte fórmula: 35 mililitros $(\mathrm{mL})$ de água dividido pelo peso em quilogramas $(\mathrm{Kg})$. Outro valor importante estimado em adultos é a água endógena, ou seja, resultante de reações químicas do nosso organismo (Ciclo de Krebs). Para exemplificar, estima-se que o volume de água endógena produzida em vinte e quatro horas por um adulto de 70 quilos, seja de $300 \mathrm{~mL}$.

$\mathrm{O}$ cálculo do $\mathrm{BH}$ em adultos nem sempre considera as perdas insensíveis, ou seja, as perdas de líquidos por meio da transpiração, respiração e água endógena, devido a impossibilidade de medir os volumes. Contudo, sabe-se que a perda de água por meio da respiração é muito grande em pacientes que necessitam de ventilação mecânica (CUNHA; LOBO, 2015).

O correto preenchimento e cálculo do $\mathrm{BH}$, requer inicialmente a definição de padrões e esclarecimento quanto aos tipos de líquidos que serão registrados. A equipe de Enfermagem da UTI deve conhecer particularidades de cada paciente, quanto aos tipos de dispositivos em uso e formas adequadas de mensuração dos volumes de líquidos desprezados (NETTO ET AL., 2015; LINHARES ET AL., 2016). 


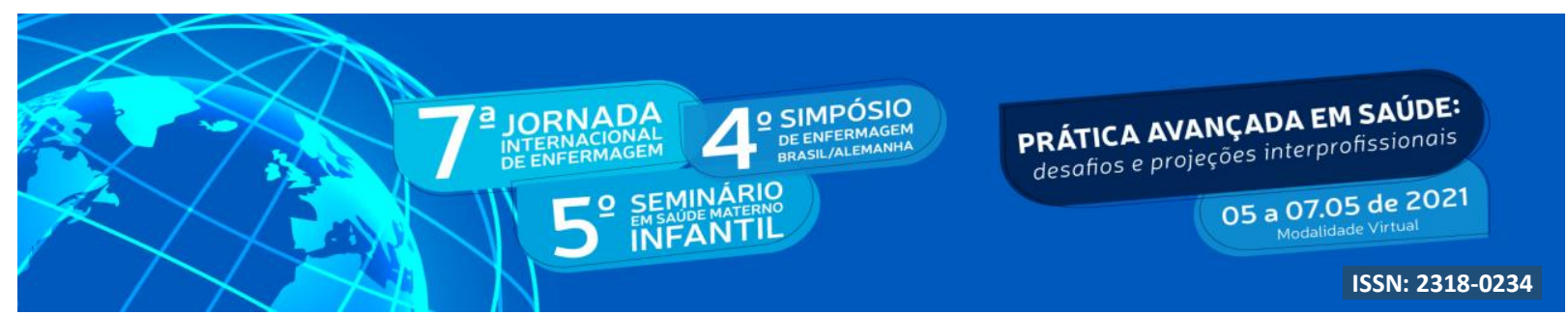

\section{Preenchimento do BH}

Na prática clínica o BH é obtido a partir de operações matemáticas simples, na qual somam-se líquidos infundidos e subtraem-se dos líquidos eliminados. Assim, o resultado preservará o sinal positivo ou negativo, conforme o maior valor obtido, ou seja, se o total de líquidos fornecidos ao paciente for maior, o sinal positivo (+) precederá o resultado do $\mathrm{BH}$ e, se o volume total de líquidos eliminados for maior, então o sinal que precederá o resultado será negativo (-).

No quadro 1, foram apresentadas as principais vias de entrada e saída de líquidos, seguido da exemplificação de dispositivos comumente utilizados em pacientes na UTI.

Quadro 1: Apresentação das vias para administração e eliminação de líquidos

\begin{tabular}{|c|c|c|c|}
\hline \multicolumn{4}{|c|}{ Entrada de líquidos (+) } \\
\hline Via & Local & Dispositivo & Exemplo \\
\hline$>$ Parenteral & $\begin{array}{l}\text { Rede } \\
\text { venosa }\end{array}$ & $\begin{array}{l}\text { Cateter venoso central } \\
\text { Cateter venoso de } \\
\text { inserção periférica }\end{array}$ & $\begin{array}{l}\text { - Hidratação venosa } \\
\text { - Hemotransfusão } \\
\text { - Drogas vasoativas }\end{array}$ \\
\hline$>$ Enteral & Sistema & $\begin{array}{l}\text { Sonda enteral } \\
\text { Sonda gástrica }\end{array}$ & $\begin{array}{l}\text { - Dieta por sonda, Gastrostomia, } \\
\text { Jejunostomia }\end{array}$ \\
\hline$>$ Oral & & - & $\begin{array}{l}\text { - Hidratação oral } \\
\text { - Dieta oral }\end{array}$ \\
\hline \multicolumn{4}{|c|}{ Saída de líquidos (-) } \\
\hline Via & Local & Dispositivo & Exemplo \\
\hline$>$ Parenteral & $\begin{array}{l}\text { Rede } \\
\text { venosa }\end{array}$ & $\begin{array}{l}\text { Cateter venoso central } \\
\text { (duplo lúmen) }\end{array}$ & - Hemodiálise \\
\hline$>$ Enteral & $\begin{array}{l}\text { Sistema } \\
\text { digestório }\end{array}$ & $\begin{array}{l}\text { Sonda enteral aberta } \\
\text { Sonda gástrica aberta }\end{array}$ & - Conteúdo gástrico \\
\hline$>$ Oral & & - & - Vômito \\
\hline$>$ Intestinal & & $\begin{array}{l}\text { Ostomias } \\
\text { Drenos }\end{array}$ & $\begin{array}{l}\text { - Conteúdo intestinal (fezes) } \\
\text { - Drenagens diversas }\end{array}$ \\
\hline
\end{tabular}




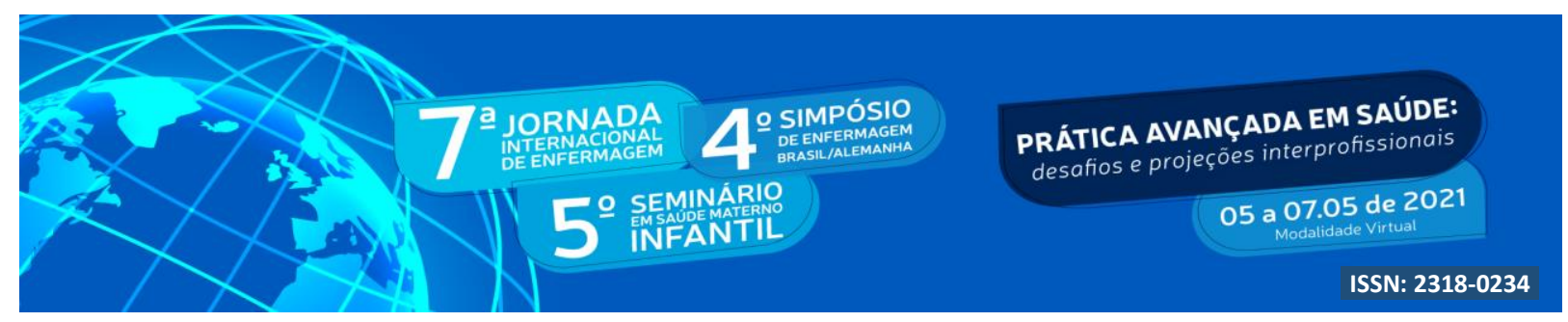

\begin{tabular}{|l|l|l|l|}
\hline$>$ Urinário & urinário & $\begin{array}{l}\text { Cateter vesical } \\
\text { Nefrostomia } \\
\text { Cistostomia }\end{array}$ & - Diurese \\
\hline & & $\begin{array}{l}\text { Dreno torácico } \\
\text { Toracocentese }\end{array}$ & $\begin{array}{l}\text { - Respiração } \\
\text { - Drenagens de líquidos } \\
\text { pulmonares }\end{array}$ \\
\hline$>$ Pulmonar & & Paracentese & - Líquido ascítico \\
\hline$>$ Pele & Sistema & & -Transpiração \\
\hline
\end{tabular}

Fonte: elaborado pelos autores, 2021.

A principal via de entrada de líquidos em pacientes críticos é a parenteral, que pode ser obtida por punção com cateter venosa periférico ou central. Os tipos de líquidos mais comumente utilizados são as soluções fisiológicas e glicosadas, com programação de reposição contínua em vinte e quatro horas. Para determinar a vazão de infusão dos liquidos parenterias serão considerados vários aspectos como, deficit de líquidos, níveis pressóricos, capacidade cardiovascular, função renal e pulmonar, entre outros (BOUCHARD; MEHTA, 2015).

Já a principal via de eliminação de líquidos é a diurese, que em pacientes crítidos é geralmente obtida por meio do cateter vesical de demora. Os coletores devem possuir sistema de marcação para visualização do volume e de características importantes como coloração e aspecto da diurese eliminada. O monitoramento do volume pode ser calculado em adultos considerando o resultado da diurese do paciente e o peso por hora $(0,5 \mathrm{~mL} / \mathrm{Kg} / \mathrm{hora})$. Este parâmetro também é adotado pela Sociedade Brasileira de Nefrologia para o acompanhamento e classificação da função renal em pacientes agudos (YU ET AL., 2007).

$\mathrm{O}$ preenchimento do $\mathrm{BH}$ é de responsabilidade da equipe de enfermagem, que deve a cada duas horas registrar os parâmetros dos sinais vitais, vazão das medicações e das reposições, volumes infundidos e eliminados (NETTO EL AL., 2015). O profissional deve registrar os volumes e após o preenchimento deve assinar o seu nome no impresso do BH.

O cálculo dos valores registrados é realizado ao meio dia, as 18 e as seis horas da 


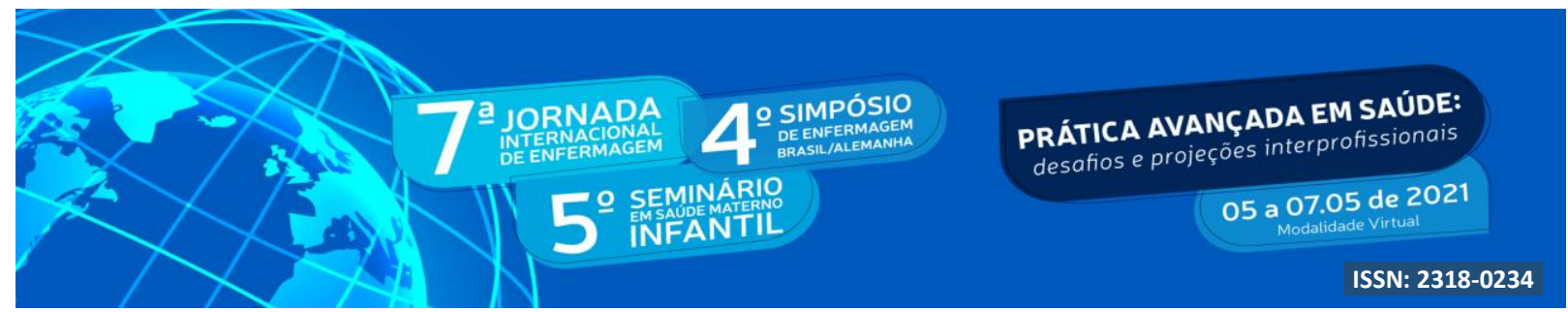

manhã. A cada passagem de plantão é importante informar os valores obtidos no BH e destacar aspectos importantes para a continuidade dos cuidados aos pacientes (MELO ET AL., 2014; NETTO ET AL., 2015).

\section{Aplicação prática do BH na UTI}

A interpretação dos valores obtidos no BH possibitam a realização de ajustes em medicações e na velocidade de infusão de fluidos, e também, serão utéis para nortear as etapas de planejamento e implementação de cuidados de Enfermagem aos pacientes no ambiente de UTI (LUCENA ET AL., 2017). Como exemplo pode-se citar: aplicar cremes de barreira para proteção da pele; reposicionar o paciente com utilização de coxins e elevar a cabeceira para facilitar a expansibilidade torácica.

$\mathrm{O}$ resultado do $\mathrm{BH}$ não deve ser analisado pontualmente e sim correlacionado a enfermidade e seus efeitos no orgnismo, parametros dos sinais vitais e evolução clínica do paciente. Durante a avaliação clínica deve-se identificar sinais de sobrecarga ou depleção de líquidos, por meio do realização do exame físico, resultados de exames laboratoriais e de imagem (LINHARES ET AL., 2016).

O BH positivo sugere retenção de líquidos, que podem ser identificados ao se avaliar os sinais vitais, especialmente na presença de frequencia cardíaca e níveis pressóricos elevados. Deve-se ainda, realizar ausculta pulmonar e cardíaca em busca de sinais de congestão e por fim, avaliar mucosas e edema em tecidos periféricos (LUCENA ET AL., 2017).

A indentificação precoce de hipervolemia é de grande utilidade para prevenir complicações como picos hipertensivos e edema agudo de pulmão e reduzir a mortalidade em UTI. Pois, o BH positivo acumulado culmina em extravazamento de líquidos para compartimentos limitados como o espaço intersticial, ocasionando prejuizos na circulação sanguínea e perfusão tecidual (ÁVILA ET AL., 2014).

Estudo identificou que em pacientes com $\mathrm{BH}$ positivo o desmame da ventilação mecânica teve menor êxito, e houve também, maior número de retorno de pacientes para ventilação entre aqueles que mantiveram BH positivo nas primeiras 24 horas após extubação. Esses achados podem ser justificados em parte pela diminuição da complacência pulmonar e 


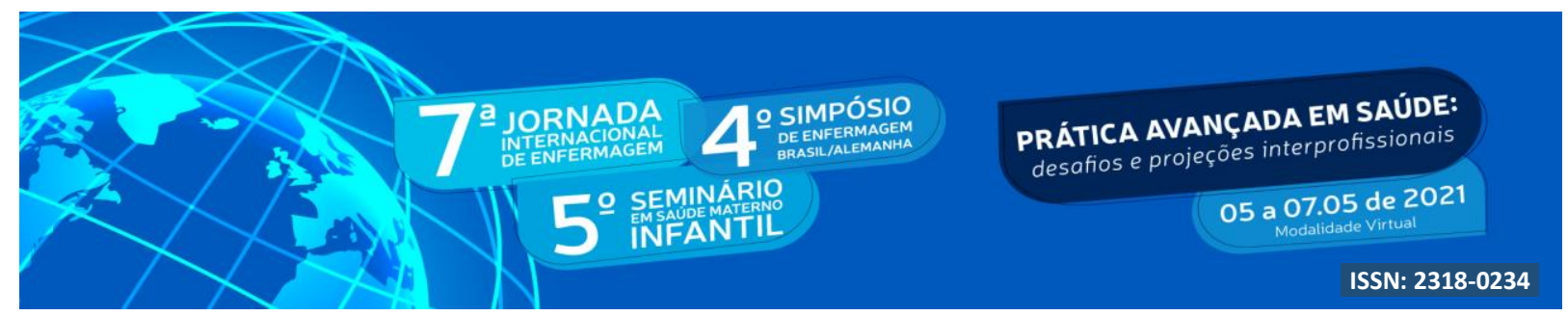

troca de gases prejudicada em pacientes hipervolêmicos (SOUZA ET AL., 2017).

Estudos revelaram que pacientes críticos que mantiveram o $\mathrm{BH}$ persistentemente positivo, evoluíram com piora clínica e elevação das taxas de mortalidade, devido a hipertensão intra-abdominal progressiva, edema de alças intestinais, íleo paralítico e por fim síndrome compartimental abdominal. Durante essa síndrome, o volume excessivo de líquidos leva ao aumento da pressão intra-abdominal que compromete a circulação sanguínea e promove deterioração da função de órgãos vitais como fígado e rins (BOUCHARD; MEHTA, 2009; CUNHA; LOBO, 2015).

$\mathrm{O} \mathrm{BH}$ negativo é menos frequente e em geral ocorre em pacientes que evoluem com diurese maior que $2.500 \mathrm{~mL}$ em 24 horas, denominado poliúria. Dentre as causas mais frequentes se destaca o diabetes insípidus, decorrênte da ausência ou liberação insuficiente do Hormônio Antidiurético (HAD), pela hipófise (MALNIC, 2009). Nesses casos, a correção deste distúrbio pode ser corrigido por meio da reposição do hormônio.

Os profissionais da UTI devem estar atentos ao BH e sempre correlacionar os resultados obtidos as condições clínicas dos pacientes, seus exames, fármacos e reposições de líquidos em uso. Com esse entendimento, se pode afirmar que a aplicação dos resultados do BH em pacientes críticos é de grande utilidade e adjuvante ao planejamento e implementação de cuidados adequados as reais necessidades individuais.

\section{CONCLUSÃO}

$\mathrm{O}$ estudo mostrou que o preenchimento do $\mathrm{BH}$ é de responsabilidade da equipe de enfermagem que, por sua vez, deve registrar a cada duas horas os volumes de todos os líquidos infundidos (via parenteral, enteral ou oral) e eliminados (hemodiálise, vômitos, líquidos drenados, fezes e diurese) pelo paciente. Além disso, fazer corretamente o cálculo do BH parcial ao término de cada turno de trabalho.

Além disso, apontou aspectos relevantes para o preenchimento e interpretação dos resultados do balanço hídrico em pacientes críticos, com ênfase para a etapa de análise dos resultados obtidos, visando favorecer o adequado manejo e regulação dos fluidos na prática clínica, bem como o planejamento e implementação de cuidados de Enfermagem aos 


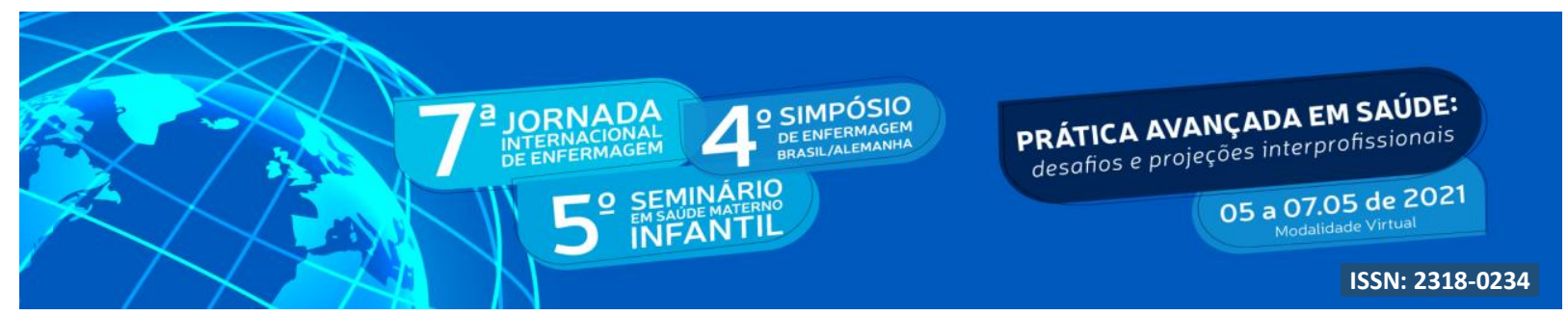

pacientes no ambiente de UTI.

\section{REFERÊNCIAS}

ÁVILA, M. O. N. ET AL. Water balance, acute kidney injury and mortality of intensive care unit patients. J. Bras. Nefrol., São Paulo, v. 36, n. 3, p. 379-388, set. 2014. [Citado em 06 dez 2020]. Disponível em http://www.scielo.br/scielo.php?script=sci_arttext\&pid=S0101$\underline{28002014000300379 \& \operatorname{lng}=\mathrm{pt} \& \mathrm{nrm}=\mathrm{iso}}$

BOUCHARD, J. MEHTA, R. L. Fluid accumulation and acute kidney injury: consequence or cause. Curr Opin Crit Care, v. 15, p. 509-13, 2009. [Citado em 21 dez 2020]. DOI: http://dx.doi.org/10.1097/MCC.0b013e328332f653

CUNHA, A. R. L.; LOBO, S. M. A. O que ocorre com o balanço hídrico durante e após a reversão do choque séptico? Rev. bras. ter. intensiva, São Paulo, v. 27, n. 1, p. 10-17, Mar. 2015. [Citado em 15 dez 2020]. Disponível em: http://www.scielo.br/scielo.php?script=sci_arttext\&pid=S0103$\underline{507 X 2015000100010 \& \operatorname{lng}=\text { en \&nrm=iso }}$

ESPINOSA-ALMANZA, C. J. ET AL. Fluid overload in patients with septic shock and lactate clearance as a therapeutic goal: a retrospective cohort study. Rev. bras. ter. Intensiva, v. 32, n. 1, p. 99-107, 2020. [Cited on 05 Mar 2021]. Available from:

http://www.scielo.br/scielo.php?script=sci_arttext\&pid=S0103$\underline{507 X 2020000100099 \& \operatorname{lng}=\text { pt\&nrm }=\text { iso }}$

GOMES, P. P. S. ET AL. Balanço hídrico na nefrologia pediátrica: construção de um Procedimento Operacional Padrão. Rev. Bras. Enferm., Brasília, v. 71, supl. 3, p. 1404-1411, 2018. [Citado em 05 Mar 2021]. Disponível em:

http://www.scielo.br/scielo.php?script=sci_arttext\&pid=S0034-

$\underline{71672018000901404 \& \operatorname{lng}=e n \& n r m=i s o}$

GUMS, Larissa da Silva; SOUZA, Jéssica dos Santos; SOUZA, Laurindo Pereira.

Mapeamento dos registros de balanço hídrico em uma unidade de terapia intensiva pública no interior sul da Amazônia Legal. Rev Elet Acervo Saúde, v. 13, n. 2, p. e6279, 2021. [Citado em 05 Mar 2021]. Disponível em:

https://acervomais.com.br/index.php/saude/article/view/6279

LINHARES, J. C. C. ET AL. Aplicabilidade dos resultados de enfermagem em pacientes com insuficiência cardíaca e volume de líquidos excessivo. Rev Gaúcha Enferm. v.37, n.2, p. e61554, 2016. [Citado em 15 dez 2020]. Disponível em: http://www.scielo.br/scielo.php?script=sci_arttext\&pid=S1983$14472016000200419 \& \operatorname{lng}=\mathrm{en} \& n r m=$ iso

LUCENA, A. F. ET AL. Validation of the nursing interventions and activities for patients on $9^{\text {hemodialytic therapy. Rev Gaúcha }}$ 


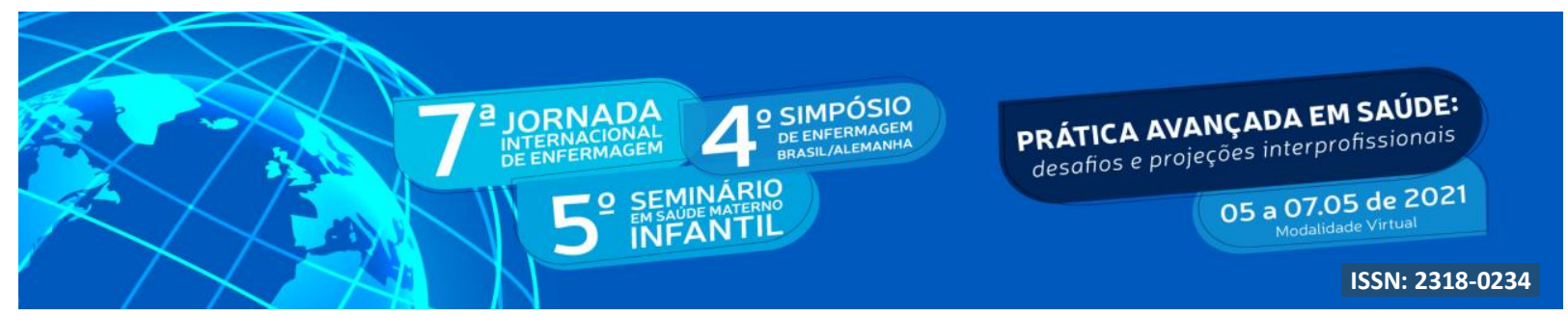

Enferm., Porto Alegre, v.38, n. 3, e66789, 2017. [Cited 05 Dec 2020]. Available from: http://www.scielo.br/scielo.php?script=sci_arttext\&pid=S1983$\underline{14472017000300402 \& \operatorname{lng}=\text { en } \& n r m=\text { iso }}$

MALNIC, G. Os fluidos biológicos. In: CURI, Ruy. Fisiologia Básica. Cap. 1, Rio de Janeiro: Guanabara Koogan, 2009, p.3-14.

MELO, E. M. ET AL. Avaliação dos registros de enfermagem no balanço hídrico de pacientes em unidade de terapia intensiva. Rev enferm UFPI; v. 9, n. 4, p. 35-41, out.-dez. 2014. [Citado em 20 dez 2020]. Disponível em: https://ojs.ufpi.br/index.php/reufpi/article/view/2092

NETTO, S. M. ET AL. Análise dos registros referentes ao balanço hídrico em unidade de terapia intensiva. Rev Enferm UFPE on line, v. 9, n. 1, p. 448-456, jan. 2015. [Citado em 20 dez 2020]. Disponível em:

https://periodicos.ufpe.br/revistas/revistaenfermagem/article/view/10358/11081

OGBU, O. C.; MURPHY, D. J.; MARTIN, G. S. How to avoid fluid overload. Current Opinion Critical Care, v. 21, n. 4, p. 315-21, 2015. [Citaded on 05 Mar 2020]. Available from: https://doi.org/10.1097/mcc.0000000000000211

POLIT, D. F.; BECK, C. T. Fundamentos de pesquisa em enfermagem: métodos, avaliação e utilização. 7.ed. Porto Alegre: Artmed, 2011.

SERKOVÁ, D; MARECKOVÁ, J. Validation of NANDA International diagnoses at an intensive care unit. Cent Eur J Nurs Midw, v.10, n.2, p. 1041-1051, 2019. [Citado em 05 dez 2020]. Disponível em: https://www.semanticscholar.org/paper/Validation-of-NANDAInternational-diagnoses-at-an-SerkovaMareckova/8b34d6fd5703cedee3cfc1d0a270597e4538bac6

SANTOS, R. P. ET AL. Non-recovery of renal function is a strong independent risk factor associated with mortality in AKI patients. Braz. J. Nephrol., São Paulo, v. 42, n. 3, p. 290299, Sept. 2020. [Citaded on 05 Mar 2020]. Available from: http://www.scielo.br/scielo.php?script=sci_arttext\&pid=S0101$28002020000300290 \& \operatorname{lng}=$ en $\& n r m=$ iso

SOUZA, J. C. ET AL. Interferência do balanço hidroeletrolítico no desfecho do desmame ventilatório. Arq Ciências da Saúde, [S.1.], v. 24, n. 3, p. 89-93, out. 2017. [Citado em 06 dez 2020]. Disponível em:

https://www.cienciasdasaude.famerp.br/index.php/racs/article/view/740.

YU, L. ET AL. Insuficiência renal aguda - Diretrizes Clínicas da Sociedade Brasileira de Nefrologia. J. Bras. Nefrol. V. 29, suppl. 1, 2007. [Citado em 15 dez 2020]. Disponível em: https://bjnephrology.org/wp-content/uploads/2019/11/jbn_v29s1dir01.pdf 